Supporting information

\title{
Hierarchical Surface Architecture of Hemodialysis Membranes for Eliminating Homocysteine Based on the Multifunctional Role of Pyridoxal 5'-phosphate
}

Peng Jiang, Yang He, Yiping Zhao, Li Chen*

State Key Laboratory of Separation Membranes and Membrane Processes, School of Materials Science and Engineering, Tiangong University, Tianjin 300387, China.

*Corresponding author. Email: tjpuchenlis@163.com

\section{The determination of molecular weights of HPG}

The molecular weight for hyperbranched polyglycerol (HPG) was calculated from ${ }^{1} \mathrm{H}$-nuclear magnetic resonance spectra $\left({ }^{1} \mathrm{H}\right.$ NMR) data, as shown in Figure S1. ${ }^{1} \mathrm{H}$ NMR (300 MHz, MeOH-d $): \delta(p p m)=7.32-7.00$ (m, 10H, aromatic), 4.10-3.96 (br, $\mathrm{OH}$ ), 3.83-3.40 (polyether backbone). By calculating the peak area ratio of the polyether backbone to the benzyl group, three types of $N, N$-Dibenzyl tris(hydroxymethyl) aminomethane initiated hyperbranched polyglycerol $\left(\mathrm{Bn}_{2}\right.$ TrisHPG) can be calculated and the molecular weight is about: $1450 \mathrm{~g} \cdot \mathrm{mol}^{-1}, 2700 \mathrm{~g} \cdot \mathrm{mol}^{-1}$ and $4200 \mathrm{~g} \cdot \mathrm{mol}^{-1}$. However, because of the high activity of amino groups, it is difficult to obtain the accurate characteristic peaks of amino acid from ${ }^{1} \mathrm{H}$ NMR data. We therefore calculated the corresponding molecular weights of $\mathrm{NH}_{2}-\mathrm{HPG}$ by converting the quality difference between benzyl and amino. The molecular weights of $\mathrm{NH}_{2}-\mathrm{HPG}$ can be determined to be about 1300,2550 and $4050 \mathrm{~g} \cdot \mathrm{mol}^{-1}$. 


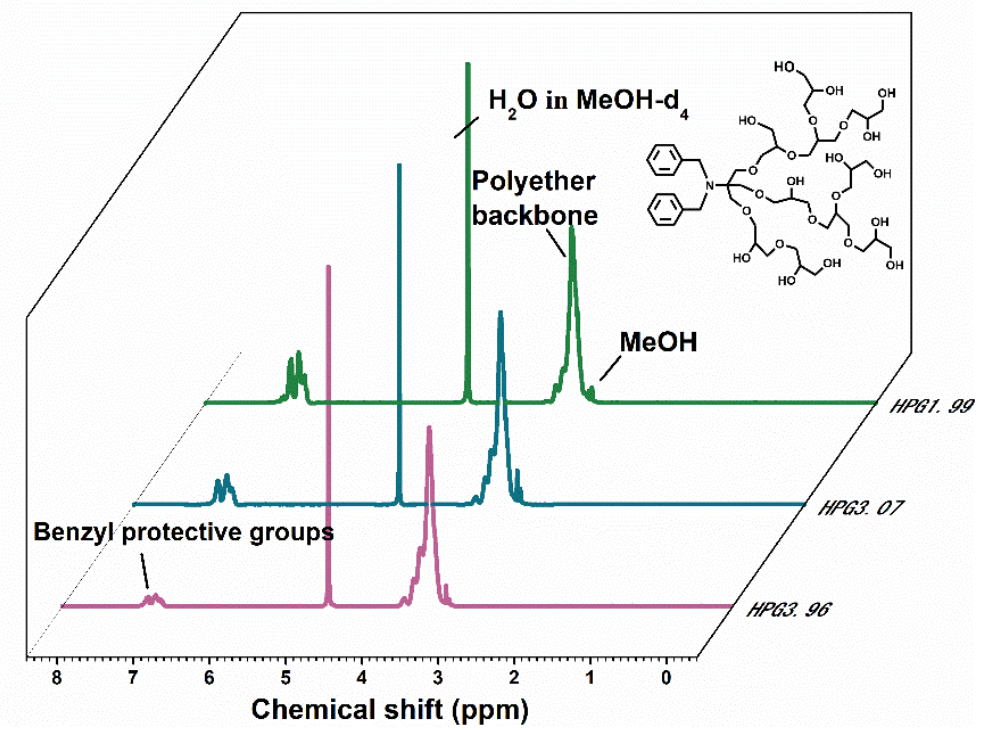

Figure S1. ${ }^{1} \mathrm{H}$ NMR spectra $\left(300 \mathrm{MHz} \mathrm{MeOH}-\mathrm{d}_{4}\right)$ of $\mathrm{Bn}_{2}$ Tris-HPG.

\section{The degree of chain volume overlap}

The physical quantity of the degree of chain volume overlap $\left(D_{v}\right)$ is first inspired by the degree of chain overlap $\left(L / 2 R_{g}\right)$, which is presented by Prof. Benetti. ${ }^{1,2}$ The degree of chain overlap $\left(L / 2 R_{\mathrm{g}}\right)$ has been applied to characterize the compactness of grafted chains, which expressed as the ratio of the distance between grafting points $(L)$ and radius of gyration of each polymer adsorbate $\left(R_{g}\right)$. However, considering the threedimensional conformation of dendritic HPG, the stretched pendant chains will provide a more shielding area beyond linear analogues and the value of $L / 2 R_{\mathrm{g}}$ is insufficient to evaluate the distribution of hyperbranched polymers comprehensively. Thus, we calculated the overlapped spatial volume between neighboring HPG chains. The schematic diagram of the calculation principle with respect to $D_{v}$ is shown in Figure $\mathbf{S 2}$ and the detail of calculation for $D_{v}$ as follows.

We first calculated the projected area of each HPG chain $\left(S_{p}\right)$ and overlapped area $\left(S_{o}\right)$ between every two adjacent HPG chains as displayed in Figure S2, and the calculated methods according to a definite integral formula, as described in the following equations: 


$$
\begin{gathered}
S_{p}=\pi R_{g}{ }^{2}(1) \\
S_{O}=4 \int_{0}^{x} \sqrt{2 R_{\mathrm{g}} x-x^{2}} d x
\end{gathered}
$$

where $x=R-R \cdot \cos \alpha, \quad \alpha=\arccos (L / 2 R)$, and thus, the Eq (2) can be equivalent to the following equation:

$$
S_{\mathrm{o}}=4 \int_{0}^{\operatorname{arc} c o s\left(\frac{L}{2 R}\right)} R \sin \partial \sqrt{R^{2}-R^{2} \cos ^{2} \partial} d \partial
$$

we can consequently get a ratio of overlapped area and projected area of single HPG chain (i.e. $D_{s}=S_{o} / S_{p}$ ). On the basis of calculated $D_{s}$, we can acquire the degree of chain volume overlap by presuming the occupied volume of individual HPG is constituted by a hemispheroid and a cone.

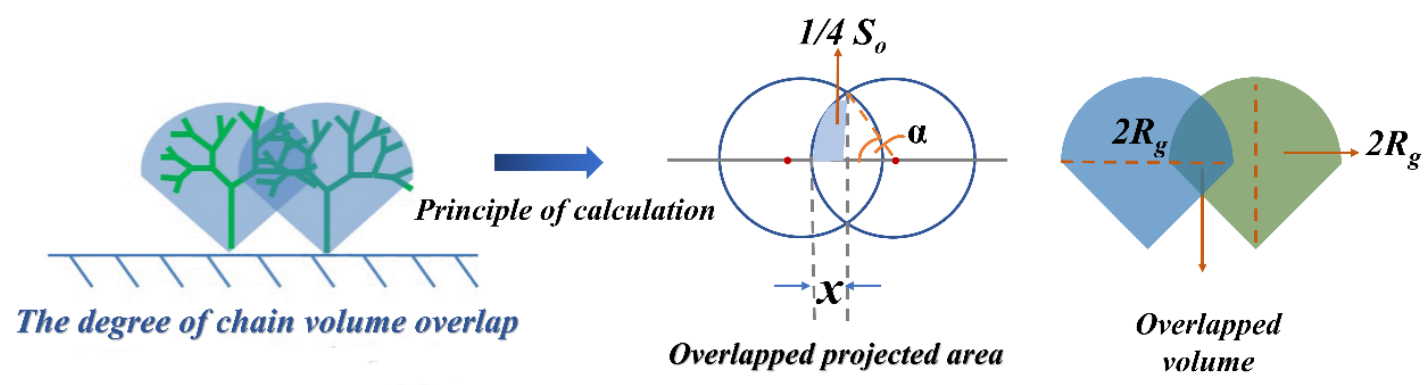

Figure S2. The illustration for the degree of chain volume overlap $\left(D_{v}\right)$ and the relevant schematic diagram of calculation principle.

\section{Plasma collection}

Fresh blood was collected from a healthy adult (male, 23 years old) utilizing anticoagulant-contained vacuum tubes to be collected according to the ratio of an anticoagulant: blood is 1:9. Platelet-rich plasma (PRP) was acquired via putting the whole blood into a centrifuge and centrifuged at $1000 \mathrm{rpm}$ for $20 \mathrm{~min}$ at $4{ }^{\circ} \mathrm{C}$. Plateletpoor plasma (PPP) was attained by centrifuging the blood at $4{ }^{\circ} \mathrm{C}$ sustained $15 \mathrm{~min}$, revolving speed set as $3000 \mathrm{rpm}$. Red blood cells (RBCs) were separated from the whole blood in the same separation way as obtaining PPP and is distinguished by removing the supernatant of PPP, the PBS ( $\mathrm{pH}=7.4$ ) was used to wash bottom fluids, after washing three times, RBCs were obtained. 


\section{Platelet adhesion}

The membrane samples $\left(1 \times 1 \mathrm{~cm}^{2}\right)$ were washed with PBS several times subsequently soaked in PBS (10 mL, pH 7.4) to equilibrate for $30 \mathrm{~min}$ and kept in the circumstances maintained a constant temperature at $37^{\circ} \mathrm{C}$. The membranes were placed in a 24-well culture plate and immersed in PRP to incubate for 60 min at $37^{\circ} \mathrm{C}$. Then, PRP was wiped off and the membranes were rinsed by PBS three times. The adherent platelets on resultant membranes surface were fixed through immersing in $2 \mathrm{~mL} 2.5 \mathrm{wt} \%$ glutaraldehyde for $24 \mathrm{~h}$ at $4{ }^{\circ} \mathrm{C}$. Lastly, the membranes were dehydrated by a series of concentration graded alcohol-PBS solution $(25 \%, 50 \%, 75 \%, 85 \%, 90 \%, 95 \%$, and $100 \%$ ). The adhesion of platelets was observed by TFE-SEM (ZEISS Gemini SEM500).

\section{Red cell adhesion}

The membrane samples $\left(1 \times 1 \mathrm{~cm}^{2}\right)$ were firstly rinsed with PBS several times, and then soaked in PBS (10 mL, pH 7.4), subsequently, placed it in a constant temperature environment for $30 \mathrm{~min}$ at $37^{\circ} \mathrm{C}$. The membranes were soaked in red blood cells to incubate for $60 \mathrm{~min}$ at $37^{\circ} \mathrm{C}$. The following steps were executed in the same manner as described above of platelet adhesion (see details at section 2).

\section{The stability of PLP coating and PLP-HPG layers functionalized membranes}

In order to verify the bonding strength between PLP and membrane surfaces and the stability of functional layers, we tested the variation in mass and hydrophilicity of PLP coating or PLP-HPG layers modified PES membranes before and after rinsed by deionized water. The modified membranes $\left(12 \times 5 \mathrm{~cm}^{2}\right)$ were assembled into the customized dialyzer for plat membranes and convection subsequently flowed through the upper and lower surfaces of membranes. The flow rate is consistent with the dialysis simulation experiment (200 and $500 \mathrm{~mL} / \mathrm{min}$ for the flow above and below the membrane, respectively). We tested the mass changes of the functional coating on membranes after rinsed for $12 \mathrm{~h}$ and $48 \mathrm{~h}$ (Table S1) using a precision balance with an accuracy of $1 \times 10^{-5} \mathrm{~g}$ (ALC-210.4, Sartorius, Germany), and the relevant hydrophilicity 
was characterized by water contact angle (Figure S3).

Table S1. The variation in deposition density $\left(\mu \mathrm{g} / \mathrm{cm}^{2}\right)$ of PLP coating and PLP-HPG layers before and after rinsed by deionized water.

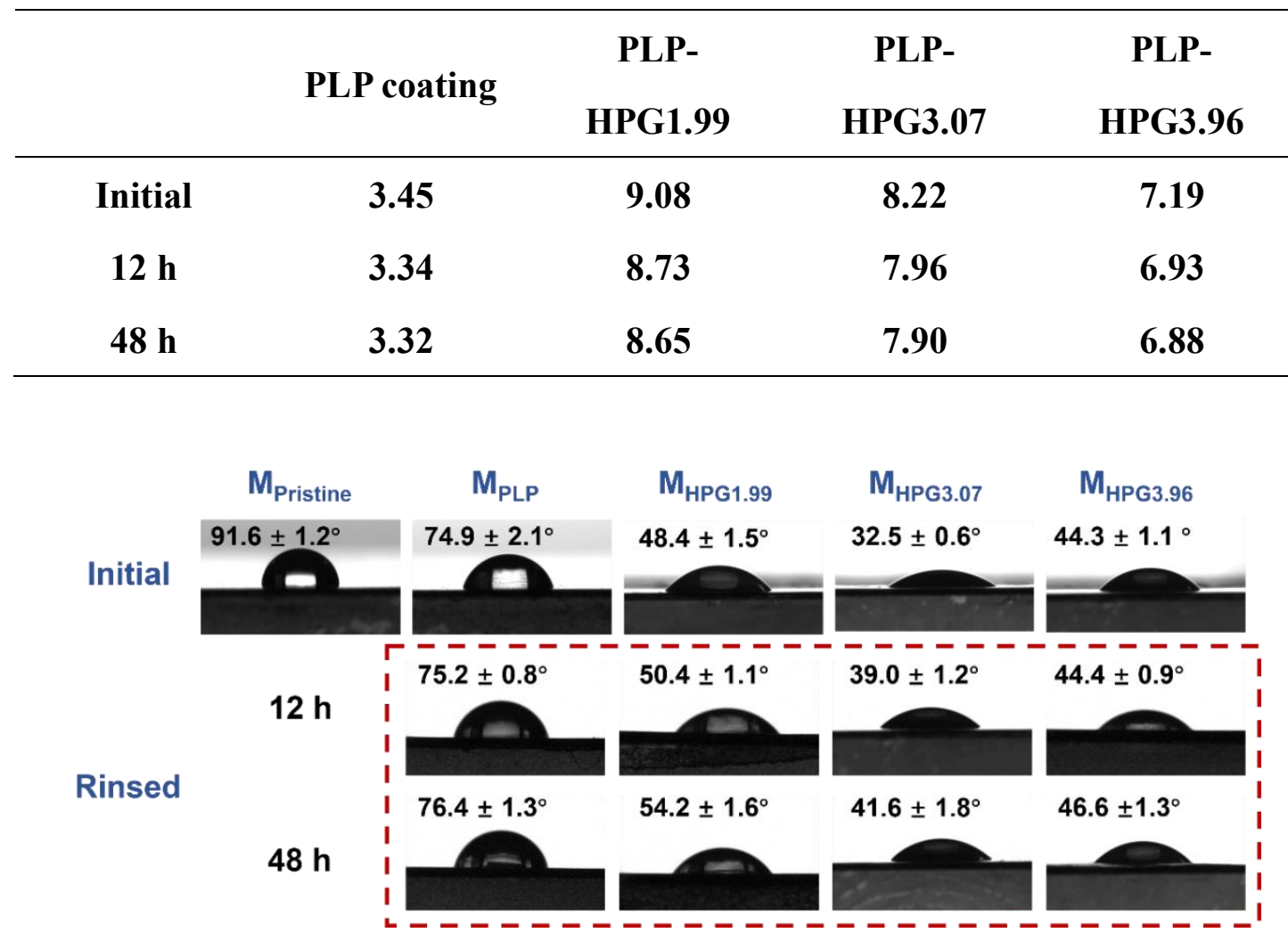

Figure S3. Pictures and static water contact angles of the modified PES membrane surfaces are shown before (top row) and after rinsed by deionized water (two bottom rows).

\section{Long-term properties of PLP-HPG layers functionalized membranes for eliminating homocysteine and resisting adsorbed proteins}

For ensuring the stability and the long-term performance of PLP-HPG layers modified PES membranes, we tested the relevant properties of the reused modified membranes (M $\mathrm{M}_{\mathrm{HPG} 1.99}, \mathrm{M}_{\mathrm{HPG} 3.07}$ and $\left.\mathrm{M}_{\mathrm{HPG}} .96\right)$ with respect to the conversion efficiency of Hcy and the antifouling ability towards proteins in 4 months later after the initial use. Figure S4a-c demonstrate the HPLC chromatograms of the initial Hcy stock solution and the Hcy-containing solution after the simulated dialysis using PLP-HPG layers modified PES membranes. It obvious that the Hcy concentration significantly 
decreased after dialysis judging from the chromatogram of different samples. The concentration of Hcy stock solution is approximately $150 \mu \mathrm{M}$, and after dialysis using PLP-HPG layers modified PES membranes, the concentration of Hcy almost all dropped below $20 \mu \mathrm{M}$ whether the membrane is used for the first time or reused after 4 months. It is important to emphasize that the membranes stored for over four months after initial use still showed the excellent ability for eliminating Hcy, although there were slight increases of Hcy not cleared by dialysis (the concentration of Hcy from 15.4 $\mu \mathrm{M}$ increased to $20.6 \mu \mathrm{M}$ for $\mathrm{M}_{\mathrm{HPG} 1.99}, 13.6 \mu \mathrm{M}$ increased to $14.5 \mu \mathrm{M}$ for $\mathrm{M}_{\mathrm{HPG} 3.96}$ and $9.4 \mu \mathrm{M}$ increased to $12.3 \mu \mathrm{M}$ for $\mathrm{M}_{\mathrm{HPG} 3.07}$ ). These results proof the excellent using stability of modified membranes prepared in our work, and it also can be further verified by the data of the antifouling properties demonstrated via the AFM adhesion curves (Figure S4d-f). Here, we selected human serum albumin (HSA, $66.5 \mathrm{kDa}$, J \& K Scientific, Beijing, China) as a model of biological contaminant. Comparing the results of the adhesion between the membranes and HSA, the values of the adhesive force of the membranes stored for over 4 months merely got little increase (no more than 10 $\mathrm{nN})$. Hence, it can be concluded that the PLP-HPG layer modified membranes are fully capable of maintaining long-term and well resistance against proteins.

(a)

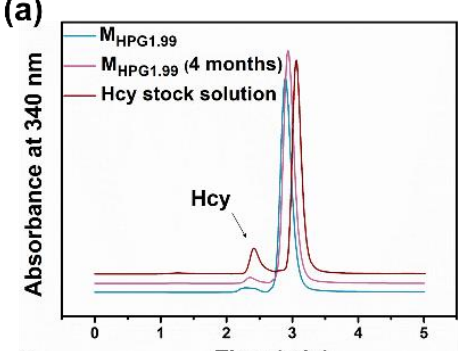

(d)

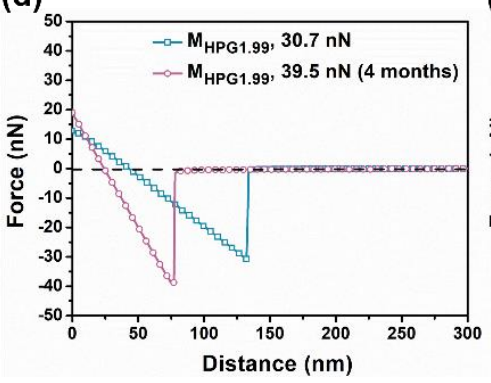

(b)

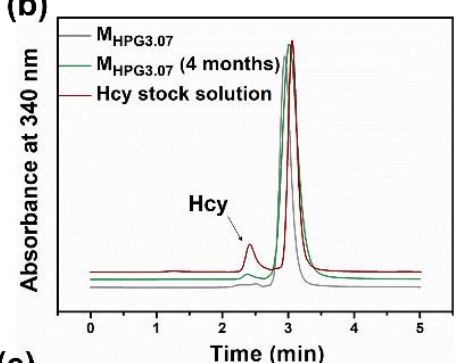

(e)

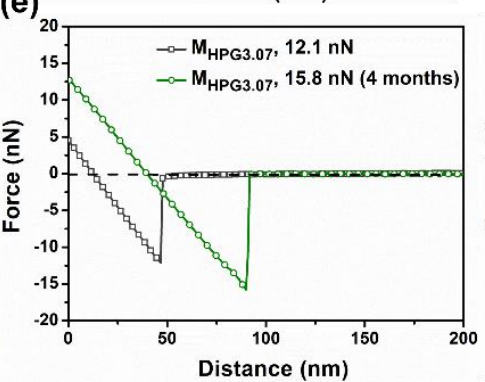

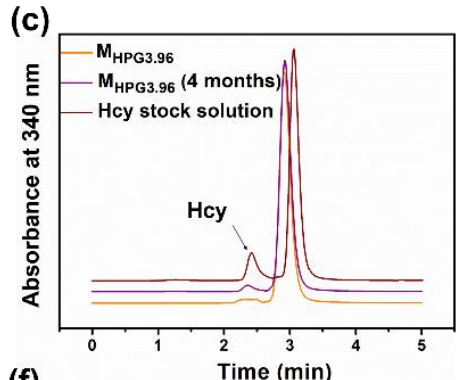

(f)

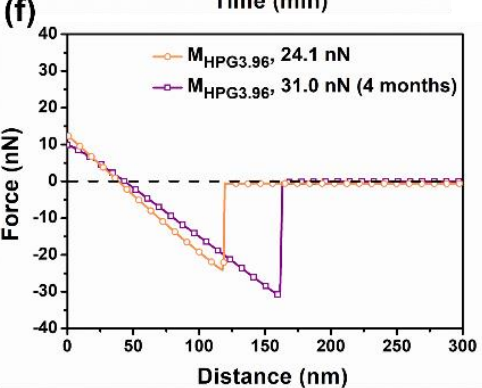

Figure S4. The long-term properties of PLP-HPG layers modified PES membranes with respect to the conversion efficiency of Hcy (a-c) and antifouling ability toward proteins (c-e). (a), (d): MHPG1.99; (b), (e): MHPG3.07; (c), (f): MHPG3.96. 


\section{References}

(1) Divandari, M.; Morgese, G.; Ramakrishna, S. N.; Benetti, E. M. Surface-Grafted Assemblies of Cyclic Polymers: Shifting between High Friction and Extreme Lubricity. Eur. Polym. J. 2019, 110, 301-306.

(2) Divandari, M.; Trachsel, L.; Yan, W.; Rosenboom, J.-G.; Spencer, N. D.; ZenobiWong, M.; Morgese, G.; Ramakrishna, S. N.; Benetti, E. M. Surface Density Variation within Cyclic Polymer Brushes Reveals Topology Effects on Their Nanotribological and Biopassive Properties. ACS Macro Lett. 2018, 7 (12), 1455-1460. 